\title{
Was verdienen Ingenieure und Ingenieurinnen? Eine Analyse auf Basis der WSI-Lohnspiegel- Datenbank
}

\author{
Fikret Öz \\ Reinhard Bispinck
}

Berufsbezogene Einkommensanalysen stoßen auf ein breit gefächertes Interesse: Die Beschäftigten nutzen sie etwa zur Orientierung bei der Arbeitsplatzsuche und bei individuellen Gehaltsverhandlungen, die Unternehmen greifen auf sie bei ihrer betrieblichen Vergütungspolitik zurück, und auch die Tarifparteien nutzen sie für die Aushandlung bzw. Anpassung von Entgeltstrukturen. Seit einigen Jahren verfügt das WSI mit der Lohnspiegel-Datenbank über eine eigene Datenbasis, die auf einer ständigen Online-Erhebung basiert. Dieser Beitrag beschreibt am Beispiel der Berufsgruppe der Ingenieure einige Möglichkeiten der vertieften berufsbezogenen Einkommensanalyse.

\section{Projekt und Daten- grundlage}

Das Projekt „Lohnspiegel“ hat die Erhebung und Analyse von Einkommens- und Arbeitsbedingungen von Beschäftigten in Deutschland zum Gegenstand. Es ist Bestandteil des internationalen Wage-Indicator-Netzwerks (www.wageindicator. org), an dem Projekte aus insgesamt 60 Ländern mit gleicher Zielrichtung beteiligt sind. Das Projekt Lohnspiegel wird vom Wirtschafts- und Sozialwissenschaftlichen Institut in der Hans-Böckler-Stiftung durchgeführt (Bispinck et al. 2010).

Die Lohnspiegel-Daten werden im Rahmen einer kontinuierlichen OnlineErhebung ermittelt, an der sich die Besucher der Webseite „www.lohnspiegel.de“ freiwillig und anonym beteiligen können. Zurzeit bietet der Lohnspiegel einen Online-Gehalts-Check für rund 280 Berufe.

Seit Ende 2004 haben rund 180.000 Beschäftigte den deutschen Online-Fragebogen ausgefüllt. Das Datenmaterial des Lohnspiegels ermöglicht Analysemöglichkeiten zu den Einkommens- und Arbeitsbedingungen der Befragten sowie ihrer darauf bezogenen Lebenseinstellungen, Präferenzen und Einschätzungen. ${ }^{1}$

Dieser Bericht analysiert sieben verschiedene Ingenieurberufe: Bauingenieu- re, Chemieingenieure, Elektroingenieure, Elektronik- und Fernmeldeingenieure, Maschinenbauingenieure, Softwareingenieure und Wirtschaftsingenieure. Die anderen Fachrichtungen werden zur Gruppe der sonstigen Ingenieurberufe zusammengefasst. Insgesamt liegen zu diesen Berufen rund 11.000 Datensätze vor, die von 2006 bis Mitte 2010 erhoben wurden.

\section{2 \\ Einkommensanalyse}

\subsection{MITTLERE VERDIENSTE}

Ingenieurberufe zählen zu den relativ hoch bezahlten Berufen. Ein Vergleich mit anderen Berufen zeigt, dass sich die Monatsverdienste ${ }^{2}$ der Ingenieure auf der obersten Einkommensebene befinden. Im Durchschnitt verdienen Ingenieure monatlich $4.380 €$. Lediglich drei Berufe in unserem Datensatz verfügen über ein höheres Monatseinkommen.

Eine genauere Differenzierung zeigt: Der Medianwert beträgt für Ingenieure $4.125 €$. Das heißt $50 \%$ der Fälle erhalten mehr und $50 \%$ der Fälle weniger als diesen Betrag. In Tabelle 1 werden auch 25-\%- und 75-\%-Perzentile dargestellt. Diese Quartile unterteilen die Verteilung in vier gleich große Gruppen. Das
75-\%-Perzentil gibt an, dass drei Viertel der Befragten ein monatliches Bruttoeinkommen von weniger als $5.221 €$ haben, ein Viertel dagegen mehr.

Das Einkommen in den einzelnen Ingenieurberufen unterscheidet sich sehr stark. Während ein Elektronik- und Fernmeldeingenieur monatlich im Durchschnitt $4.836 €$ verdient, fällt dieser Betrag bei den Bauingenieuren mit $3.709 €$ deutlich geringer aus. Zu berücksichtigen sind auch die Medianwerte, die auf die

1 Bisher liegen auf Basis der WSI-LohnspiegelDatenbank für zehn Berufe detaillierte Einkommensanalysen vor, so z. B. für Diplomkaufleute, Fachinformatiker, Kraftfahrzeugmechatroniker und IT-Systemadministratoren (Gückelhorn et al 2010a; 2010b; 2009a; 2009b) sowie für Bankkaufleute, Techniker und Bürokaufleute (Öz/ Bispinck 2009a; 2009b; 2008)

2 Unter Monatsverdienst wird hier das auf 40 Wochenstunden standardisierte regelmäßige Bruttomonatsgehalt verstanden (ohne Sonderzahlungen wie Urlaubs- und Weihnachtsgeld bzw. Jahresgratifikation)

Fikret Öz, ist Wissenschaftler im Institut Arbeit und Technik (IAT).

e-mail: oez@iat.eu

Reinhard Bispinck, Dr., ist Wissenschaftler im WSI und Leiter des WSI-Tarifarchivs in der Hans-Böckler-Stiftung. e-mail: Reinhard-Bispinck@boeckler.de 


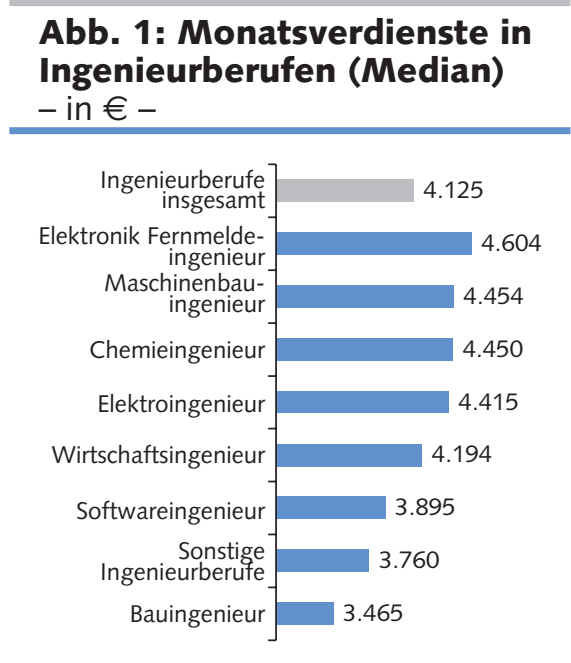

Quelle: WSI-Lohnspiegel-Datenbank - www.lohnspiegel.de.

WSI MITTEILUNGEN

Verteilung innerhalb der einzelnen Berufe hindeuten (Abbildung 1). Gemessen am Median weisen Elektronik- und Fernmeldeingenieure wiederum die höchsten
Tabelle 2: Monatseinkommen nach Betriebsgröße - in € -

\begin{tabular}{lccc}
\hline Elektronik-, Fernmeldeingenieur & $\begin{array}{c}\text { unter 100 } \\
\text { Beschäftigte }\end{array}$ & $\begin{array}{c}\mathbf{1 0 0 - 5 0 0} \\
\text { Beschäftigte }\end{array}$ & $\begin{array}{c}\text { über } \mathbf{5 0 0} \\
\text { Beschäftigte }\end{array}$ \\
Elektroingenieur & 3.953 & 4.671 & 5.312 \\
Chemieingenieur & 3.933 & 4.517 & 5.067 \\
Maschinenbauingenieur & 4.126 & 4.523 & 5.049 \\
Wirtschaftsingenieur & 3.968 & 4.574 & 4.987 \\
Sonstige Ingenieurberufe & 4.079 & 4.148 & 4.786 \\
Softwareingenieur & 3.403 & 4.127 & 4.715 \\
Bauingenieur & 3.726 & 3.891 & 4.510 \\
Ingenieurberufe insgesamt & 3.433 & 3.887 & 4.245 \\
Quelle: WSI-Lohnspiegel-Datenbank - www.lohnspiegel.de. & 3.707 & 4.322 & 4.877 \\
\hline
\end{tabular}

Einkommenswerte auf. $50 \%$ der Befragten in dieser Berufsgruppe bekommen über $4.604 €$.

\subsection{INGENIEUREINKOMMEN NACH BRANCHEN}

Fast die Hälfte der Befragten unseres Samples arbeitet in den drei Kernbranchen: Maschinenbau, Büromaschinen/Daten-
Tabelle 1: Monatsverdienst in Ingenieurberufen (40 Wochenstunden) - in $€-$

\begin{tabular}{lrrrrc}
\hline & Anzahl & Mittelwert & 25. Perzentil & Median & 75. Perzentil \\
\hline Wirtschaftsingenieur & 1.170 & 4.507 & 3.338 & 4.194 & 5.408 \\
Softwareingenieur & 1.034 & 4.067 & 3.300 & 3.895 & 4.650 \\
Sonstige Ingenieurberufe & 1.932 & 4.029 & 2.959 & 3.760 & 4.873 \\
Elektroingenieur & 1.685 & 4.660 & 3.549 & 4.415 & 5.524 \\
Elektronik-, Fernmeldeingenieur & 507 & 4.836 & 3.666 & 4.604 & 5.894 \\
Maschinenbauingenieur & 3.045 & 4.657 & 3.544 & 4.454 & 5.543 \\
Chemieingenieur & 327 & 4.668 & 3.518 & 4.450 & 5.524 \\
Bauingenieur & 1.172 & 3.709 & 2.829 & 3.465 & 4.331 \\
Ingenieurberufe gesamt & 10.872 & 4.380 & 3.293 & 4.125 & 5.221 \\
\hline & & & & WS & MITTEILUNGEN \\
Quelle: WSI-Lohnspiegel-Datenbank-www.lohnspiegel.de. & & & & &
\end{tabular}

\section{Abb. 2: Wie viel verdienen Ingenieure in den Branchen?}

$$
- \text { in } €-
$$

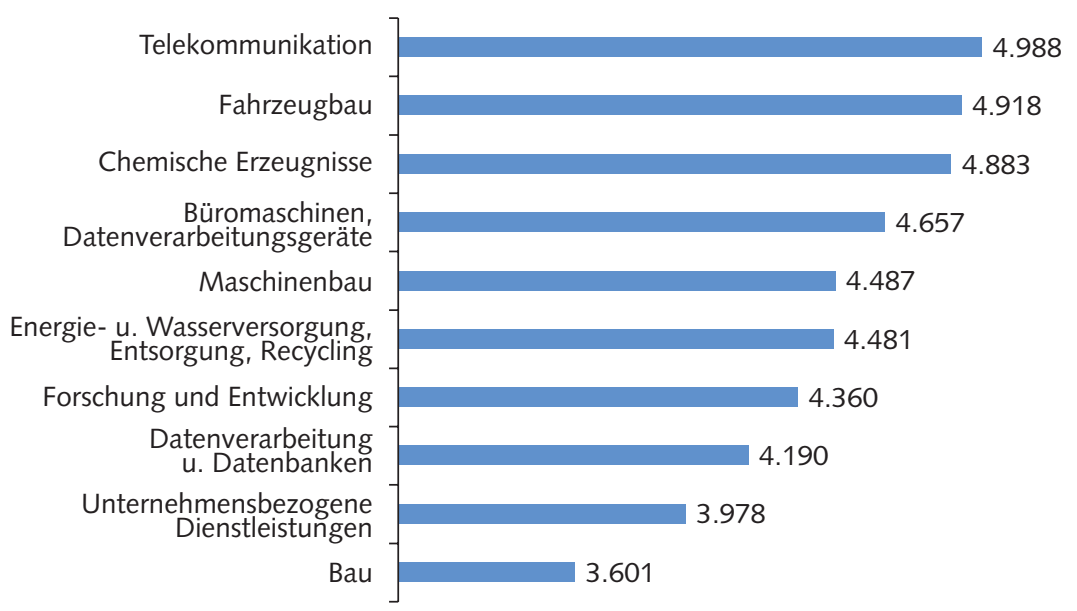

verarbeitungsgeräte und -einrichtungen sowie Fahrzeugbau. Wie unterschiedlich die Ingenieure in den Branchen bezahlt werden, zeigt Abbildung 2. Die Spanne reicht von durchschnittlich $3.601 € \mathrm{im}$ Baugewerbe über $4.487 €$ im Maschinenbau bis zu $4.988 €$ in der Telekommunikationsbranche.

\subsection{BETRIEBSGRÖSSE}

Wie zu erwarten war, ist auch bei den Ingenieuren das Einkommen stark von der Betriebsgröße abhängig (Tabelle 2). In Kleinbetrieben mit unter 100 Beschäftigten erhalten sie im Schnitt $3.707 €$, in Betrieben mit 100 bis 500 Beschäftigten sind es bereits $4.322 €$ und in Betrieben über 500 Beschäftigte werden den Ingenieuren gut $4.877 €$ gezahlt. Die Ingenieure in Großbetrieben mit über 500 Beschäftigten verdienen im Durchschnitt rund 32\% mehr als ihre Kollegen in den Betrieben mit unter 100 Beschäftigten.

\subsection{REGIONALE UNTERSCHIEDE}

\section{EINKOMMENSABSTAND WEST-OST}

Deutlich tritt der Einkommensabstand zwischen West- und Ostdeutschland in den einzelnen Ingenieurberufen zutage. Während in Westdeutschland ein Maschinenbauingenieur durchschnittlich $4.529 €$ erhält, bekommt er in Ostdeutschland $3.503 €$. Wie in Abbildung $3 \mathrm{zu}$ sehen ist, ist die Differenz am deutlichsten bei den Elektro- und Maschinenbauingenieuren. Sie verdienen im Osten jeweils rund 23 bis $24 \%$ weniger als im Westen. Am geringsten ist der Rückstand mit $14 \%$ bei den Softwareingenieuren. 


\section{EINKOMMENSABSTAND ZWISCHEN STÄDTEN}

Der Datensatz erlaubt auch Aussagen zu den Einkommensunterschieden zwischen einzelnen Städten. Das durchschnittlich höchste Einkommen für Ingenieure wird mit $4.772 €$ in Frankfurt am Main gezahlt, gefolgt von Düsseldorf mit $4.764 €$ und München mit $4.611 €$. Unterdurchschnittlich fallen die Gehälter unter anderem in Stuttgart mit $4.310 €$, Hamburg mit 4.137, Köln mit $4.101 €$ und Berlin mit $4.096 €$ aus.

Tabelle 3 zeigt neben dem Mittelwert auch unterschiedliche Quartilwerte. Dabei verändert sich zum Teil die Rangfolge der Städte. Beim 25. Perzentil, beim Medianwert und beim 75. Perzentil liegt Düsseldorf an der Spitze.

\subsection{WIRKUNGEN DER TARIFBINDUNG}

Ingenieure, die in tarifgebundenen Betrieben arbeiten, verdienen deutlich mehr als ihre Kollegen in Betrieben ohne Tarifbindung (Abbildung 4). Im Schnitt erhalten Ingenieure ohne Tarifvertrag $3.962 € \mathrm{im}$ Monat. Gilt im Betrieb ein Tarifvertrag, gibt es mit rund $4.778 €$ im Schnitt gut $816 €$ mehr Gehalt. Das entspricht knapp $21 \%$. Der Tarifeffekt wirkt in den einzelnen Ingenieurberufen unterschiedlich. Elektronik- und Fernmeldeingenieure erhalten in Betrieben mit Tarifbindung jeweils rund $12 \%$ mehr als in Betrieben ohne Tarifbindung, bei den sonstigen Ingenieurberufen sind es dagegen mehr als $23 \%$.

Neben Alter, geographischer Lage (Ost/ West) und Unternehmensgröße ist die Tarifbindung einer der wichtigsten Faktoren für die Höhe des Einkommens. In Betrieben mit Betriebsrat liegt das durchschnittliche Einkommen ebenfalls deutlich höher als in Betrieben ohne Betriebsrat.

\subsection{GEHALTSDIFFERENZ ZWISCHEN FRAUEN UND MÄNNERN}

Ingenieurberufe zählen zu den männerdominierten Berufen. Im Durchschnitt liegt in unseren Daten der Frauenanteil an den Ingenieuren bei knapp 12\%. Überdurchschnittlich ist der Anteil der Frauen bei den Chemieingenieuren mit rund $20 \%$, am niedrigsten ist er aber bei den Elektronik- und Fernmeldeingenieuren mit $3 \%$.

\section{Abb. 3: Abweichung des Ost- vom West-Verdienst}

- in $\%-$

Sonstige Ingenieurberufe

Elektroingenieur

Maschinenbauingenieur

Elektronik-, Fernmeldeingenieur

Bauingenieur

Wirtschaftsingenieur

Chemieingenieur

Softwareingenieur

insgesamt

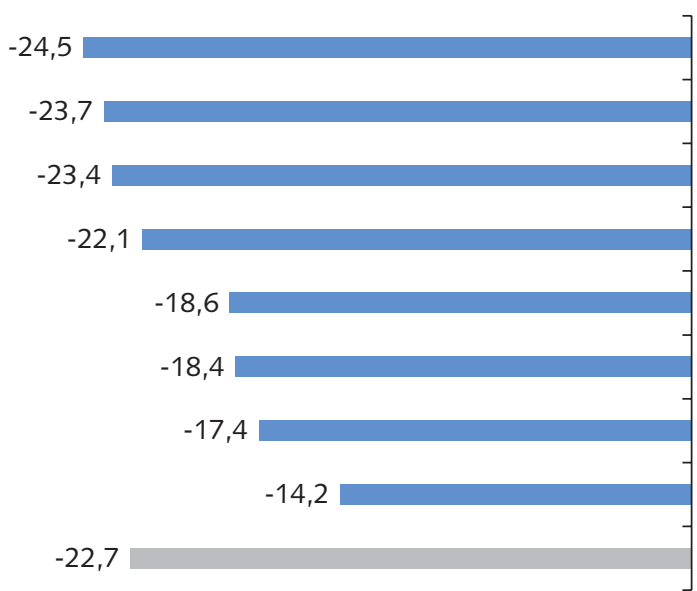

WSI mitteilungen

Quelle: WSI-Lohnspiegel-Datenbank - www.lohnspiegel.de.

der Abstand mit knapp 19\% bei den Bauingenieuren aus. In einigen Ingenieurberufen sind die Fallzahlen allerdings relativ gering, sodass die Aussagekraft der Zahlen hier begrenzt ist. zu sehen ist, verdienen Ingenieurinnen im Durchschnitt rund $17 \%$ weniger als ihre männlichen Kollegen. Noch stärker fällt
Tabelle 3: Monatsverdienst der Ingenieure in ausgewählten Städten - in $€-$

\begin{tabular}{lrrrcc}
\hline & Anzahl & Mittelwert & 25. Perzentil & Median & 75. Perzentil \\
\hline Frankfurt am Main & 89 & 4.772 & 3.562 & 4.311 & 5.410 \\
Düsseldorf & 100 & 4.754 & 3.600 & 4.563 & 5.709 \\
München & 270 & 4.511 & 3.444 & 4.188 & 5.197 \\
Stuttgart & 237 & 4.310 & 3.447 & 4.196 & 5.197 \\
Hamburg & 189 & 4.137 & 3.201 & 3.769 & 4.856 \\
Köln & 114 & 4.101 & 3.305 & 3.986 & 4.645 \\
Berlin & 268 & 4.096 & 3.035 & 3.820 & 5.099 \\
Ingenieurberufe insgesamt & 10.872 & 4.380 & 3.293 & 4.125 & 5.221 \\
\hline \multicolumn{2}{l}{ Quelle: WSI-Lohnspiegel-Datenbank - www.lohnspiegel.de. } & & & WSI MITEILUNGEN
\end{tabular}

Abb. 4: Ingenieureinkommen und Tarifvertrag - in $€-$

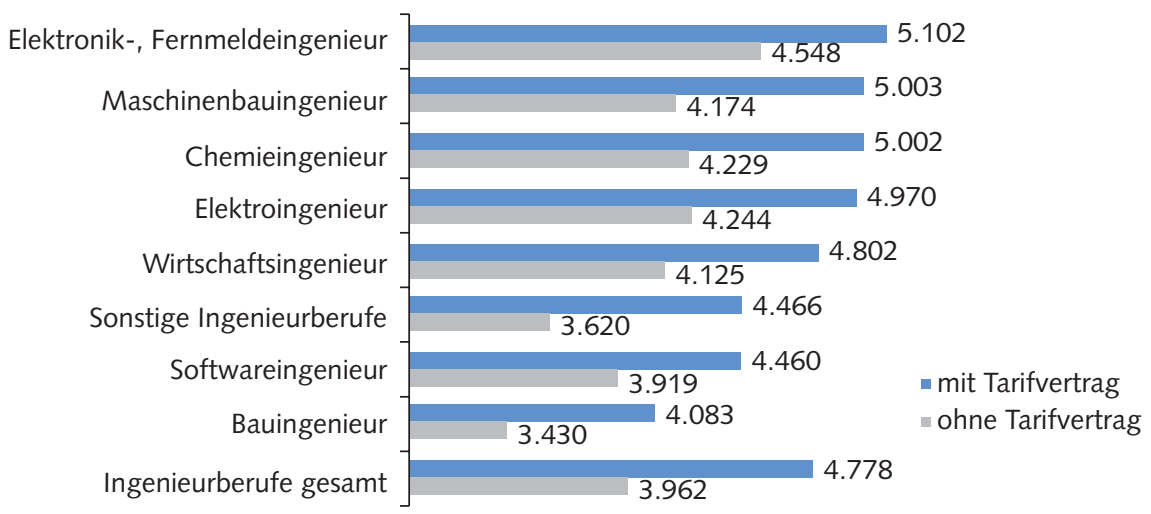

Quelle: WSI-Lohnspiegel-Datenbank - www.lohnspiegel.de. 

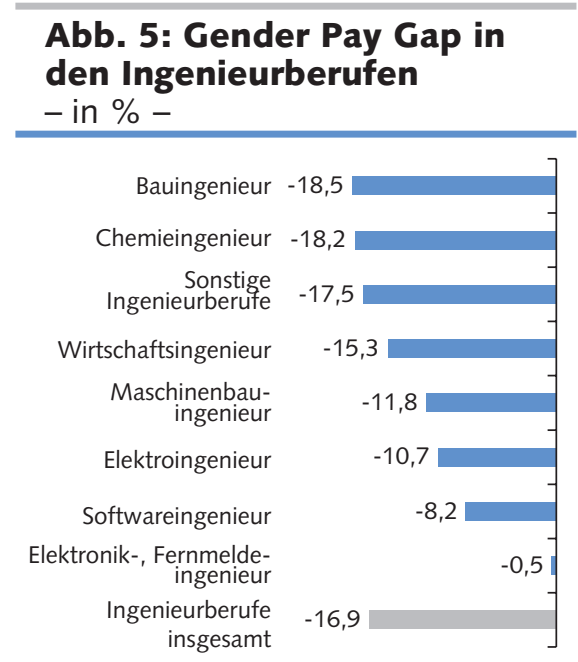

Quelle: WSI-Lohnspiegel-Datenbank - www.lohnspiegel.de. WSI MITTEILUNGEN

\subsection{BERUFSBIOGRAFISCHE ASPEKTE}

\section{AUSBILDUNGSABSCHLUSS UND EINKOMMEN}

In allen Ingenieurberufen steigt das Einkommen mit der Höhe des Ausbildungsabschlusses. Ingenieure und Ingenieurinnen mit Universitätsabschluss verdienen gut $6 \%$ mehr als ihre Kollegen mit Fachhochschulabschluss. Eine Promotion ist besonders lukrativ: Ingenieure mit Promotion erhalten nochmals rund $19 \%$ mehr als die übrigen Universitätsabsolventen. Berücksichtigt man die einzelnen Berufe, fällt diese Differenz bei den Bauingenieuren mit rund $27 \%$ besonders hoch aus. Elektronik- und Fernmeldeingenieure sowie Soft-

Tabelle 4: Berufsanfänger und Einkommensentwicklung - in $€-$

\begin{tabular}{lccc} 
& bis 1 Jahr & 2-5 Jahre & 6 und mehr Jahre \\
\hline Elektronik-, Fernmeldeingenieur & 3.615 & 3.610 & 5.155 \\
Wirtschaftsingenieur & 3.538 & 4.108 & 5.082 \\
Chemieingenieur & 3.490 & 3.895 & 5.069 \\
Maschinenbauingenieure & 3.497 & 4.015 & 5.039 \\
Elektroingenieur & 3.602 & 3.940 & 5.021 \\
Softwareingenieur & 3.421 & 3.590 & 4.416 \\
Sonstige Ingenieurberufe & 3.048 & 3.468 & 4.395 \\
Bauingenieur & 2.894 & 3.211 & 4.000 \\
Ingenieurberufe insgesamt & 3.372 & 3.771 & 4.765 \\
\hline & & WuS & MITTEILUNGEN
\end{tabular}

wareingenieure weisen dagegen auffällig niedrigere Unterschiede auf (Abbildung 6).

\section{BERUFSANFÄNGER}

Wie sich die Einstiegsgehälter für die Berufsanfänger und das Einkommen mit Berufserfahrung entwickeln, zeigt Tabelle 4. Hier werden drei Gruppen gegenübergestellt: die Berufsanfänger mit bis zu einem Jahr Berufserfahrung, mit zwei bis fünf Jahren und mit sechs und mehr Jahren. Den größten Einkommenssprung machen in den ersten fünf Jahren die Wirtschaftsingenieure mit einem Anstieg um 14\%. Bei einer Berufserfahrung von sechs und mehr Jahren können sich die Chemieingenieure mit einem Plus von rund $31 \%$ gegenüber der Einstiegsvergütung am stärksten steigern.

\section{BERUFSERFAHRUNG}

In aller Regel steigt das Einkommen mit der Dauer der Berufserfahrung. Dies gilt auch für die Berufsgruppe der Ingenieure.

\section{Abb. 6: Ingenieureinkommen nach Ausbildungsabschlüssen - in $€-$}

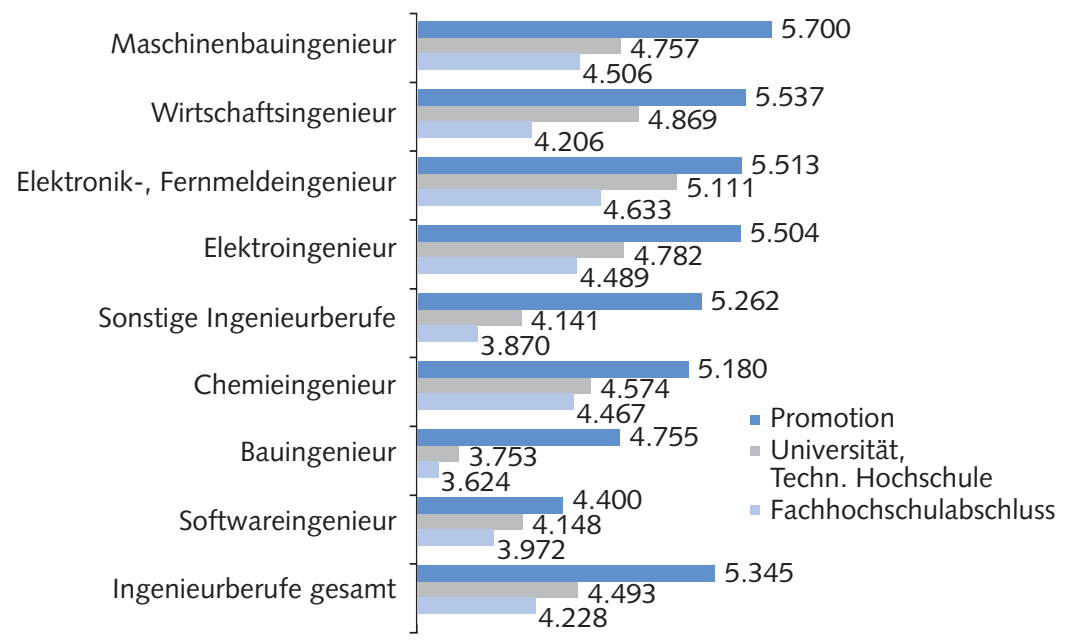

Quelle: WSI-Lohnspiegel-Datenbank - www.lohnspiegel.de.
Bei einer Berufserfahrung von unter fünf Jahren beträgt das durchschnittliche Monatseinkommen $3.626 €$, für die Gruppe mit 20 bis 30 Jahren Berufspraxis steigt es auf rund $5.000 €$. Die Anstiegsrate verlangsamt sich allerdings in den späteren Berufsjahren (Abbildung 7).

\section{BETRIEBLICHE POSITION}

Die betriebliche Position hat Einfluss auf die Höhe des Einkommens. Die Ingenieure mit Vorgesetztenposition verdienen im Schnitt $18 \%$ mehr. Die größte Differenz zeigt sich mit fast $28 \%$ bei den Wirtschaftsingenieuren, gefolgt von den Bauingenieuren mit knapp $23 \%$ und Elektroingenieuren mit 20\% (Abbildung 8).

Dies lässt sich weiter differenzieren: Von der Assistententätigkeit über Teamund Gruppenleiter bis zum Abteilungsund Hauptabteilungsleiter gibt es eine fein gestaffelte Einkommensdifferenzierung.

Frauen sind generell in den Ingenieurberufen schwach vertreten. Zwar sind Frauen auf Leitungsebenen anzutreffen,

\section{Abb. 7: Ingenieureinkommen nach Berufserfahrung - in $€-$}

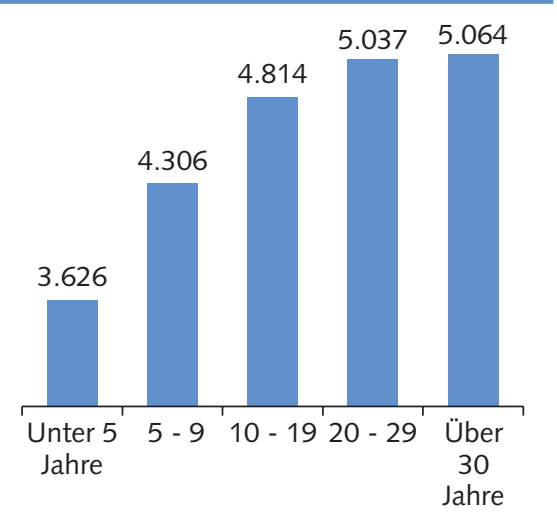

Quelle: WSI-Lohnspiegel-Datenbank - www.lohnspiegel.de. WSI MITTELUNGEN 

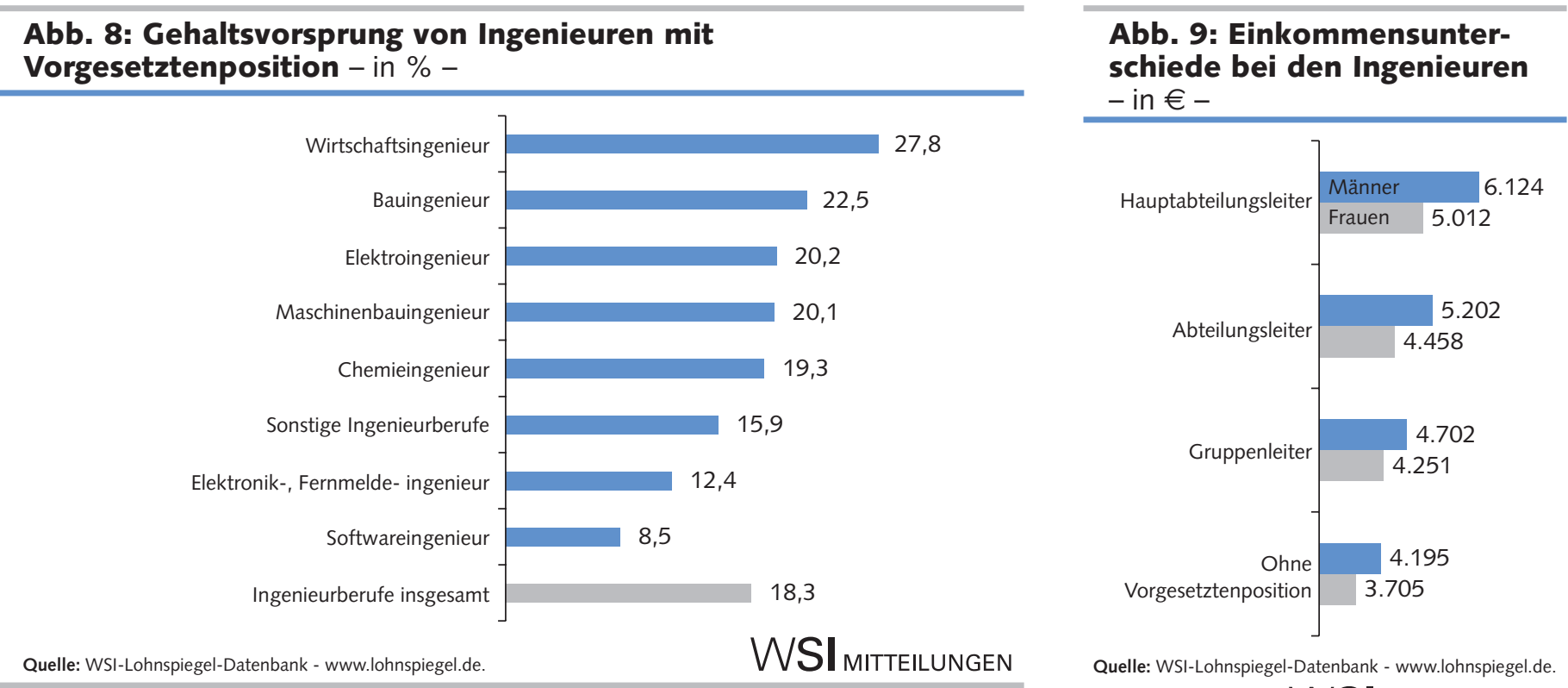

Quelle: WSI-Lohnspiegel-Datenbank - www.lohnspiegel.de.

WSI MITTELUNGEN

aber ihr Anteil bleibt sehr gering: Nur 10\% der Leitungspositionen sind von Frauen besetzt. Umso deutlicher fallen dabei die Gehaltsunterschiede aus, beispielsweise verdienen Frauen auf der Hauptabteilungsleiterposition rund $22 \%$ weniger als ihre männlichen Kollegen (Abbildung 9).

\subsection{SONDERZAHLUNGEN}

Sonderzahlungen bilden einen wichtigen Bestandteil des Verdienstes der Beschäftigten. Allerdings erhalten längst nicht alle Arbeitnehmer und Arbeitnehmerinnen eine Jahressonderzahlung, auch nicht alle Ingenieure. Wie Tabelle 5 zeigt, erhalten rund $59 \%$ der Befragten ein Weihnachtsgeld. Dieser Anteil beträgt rund $58 \%$ beim Urlaubsgeld. Weit weniger verbreitet ist die Gewinnbeteiligung. Nur rund $32 \%$ der Befragten bekommen irgendeine Form von Gewinnbeteiligung.

Positiv wirkt sich auch hier aus, wenn die Betriebe tarifgebunden sind. Während $70 \%$ der Befragten in Betrieben mit Tarifbindung Weihnachtsgeld bekommen, liegt dieser Anteil in den Betrieben ohne Tarifbindung bei $44 \%$. Eine ähnlich positive Wirkung lässt sich auch beim Urlaubsgeld feststellen.

Ein differenzierter Blick auf die Sonderzahlungen in den einzelnen Ingenieurberufen lohnt sich (Abbildung 10): Weihnachtsgeld erhalten je nach Fachrichtung zwischen 43 und $65 \%$ der Ingenieure, beim Urlaubsgeld sieht es mit 41 und $65 \%$ sehr ähnlich aus und eine Gewinnbeteiligung erhalten zwischen 20 und $36 \%$.

Tabelle 5: Sonderzahlungen bei den Ingenieuren - in \% -

\begin{tabular}{lccc}
\hline & Insgesamt & Mit Tarifbindung & Ohne Tarifbindung \\
\hline Weihnachtsgeld & 58,8 & 70,2 & 44,2 \\
Urlaubsgeld & 57,7 & 69,5 & 42,6 \\
Gewinnbeteiligung & 32,1 & 35,3 & 29,3 \\
Sonstige Sonderzahlungen & 28,3 & 30,0 & 26,9 \\
\hline \multicolumn{2}{l}{ Quelle: WSI-Lohnspiegel-Datenbank - www.lohnspiegel.de. } & & WSI MITTEILUNGEN
\end{tabular}

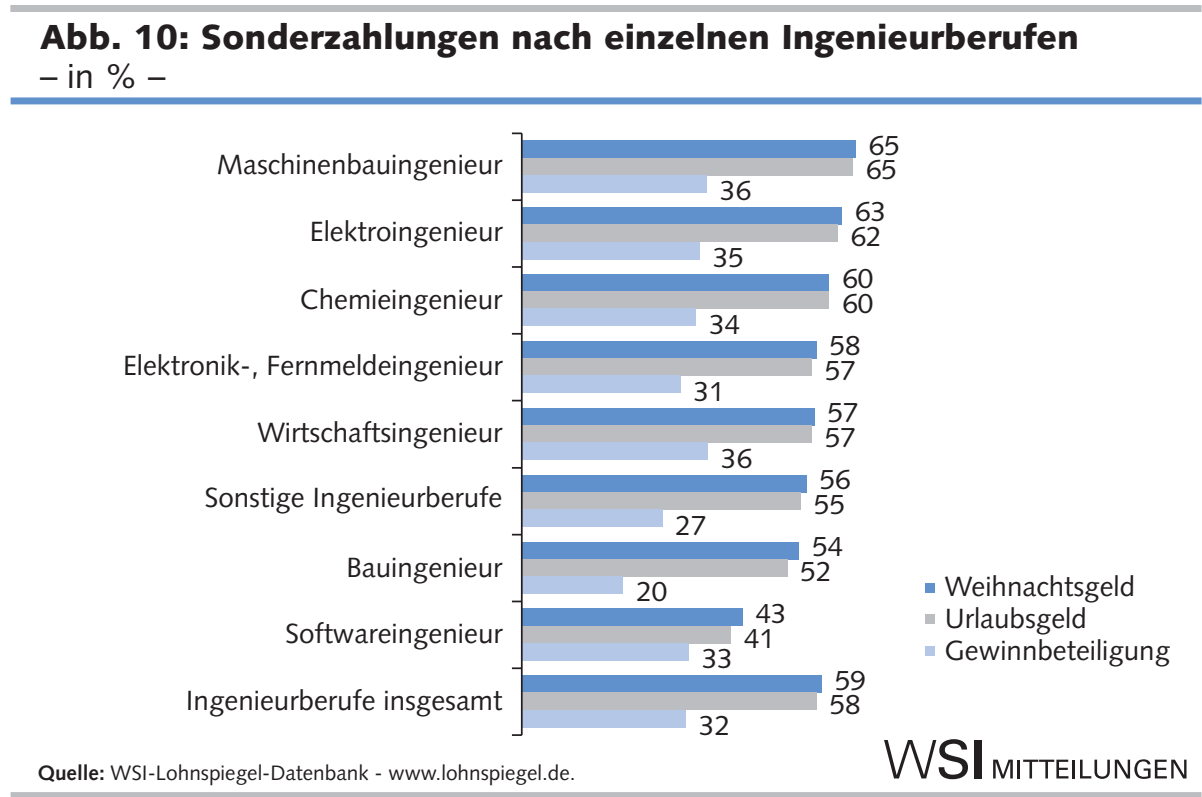

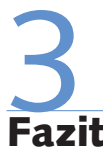

Mithilfe der Lohnspiegel-Datenbank lassen sich detaillierte Analysen der Arbeits- und
Einkommensbedingungen einzelner Berufsgruppen vornehmen. Es handelt sich aufgrund der Erhebungsmethode über eine laufende Online-Umfrage, nicht um einen repräsentativ erhobenen Datensatz. Bei entsprechend hohen Fallzahlen ergibt sich gleichwohl die Möglichkeit zu diffe- 
renzierten Auswertungen, die sowohl die betriebsbezogenen Faktoren (Branche, Betriebsgröße, regionale Lage) als auch die personenbezogenen Merkmale (Geschlecht, Ausbildung, Berufserfahrung) einschließen. Der Beitrag beschreibt Niveau und Differenzierung der Einkommen in einer Reihe von Ingenieurberufen entlang dieser Merkmale. Er bestätigt (und quantifiziert) einige Annahmen wie z.B. erheb- liche Einkommensunterschiede nach Fachrichtungen, nach Regionen und Städten, einen beachtlichen Gender Pay Gap sowie eine positive Auswirkung der Tarifbindung auf die Einkommenshöhe von Ingenieuren.

\section{LITERATUR}

Bispinck, R./Dribbusch, H./Öz, F. (2010): Das Projekt LohnSpiegel: Tatsächlich gezahlte Löhne und Gehälter, in: WSI-Mitteilungen 63 (1), S. $42-49$

Gückelhorn, C./Bispinck, R./Dribbusch, H./Öz, F. (2009a): Was verdienen Kraftfahrzeugmechatroniker und Kraftfahrzeugmechatronikerinnen? Eine Analyse von Einkommensdaten auf Basis der WSI-Lohnspiegel-Datenbank, Arbeitspapier 04, Düsseldorf Gückelhorn, C./Bispinck, R./Dribbusch, H./Öz, F. (2009b): Was verdienen IT-Systemadministratoren und IT-Systemadministratorinnen? Eine Analyse von Einkommensdaten auf Basis der WSI-LohnspiegelDatenbank, Arbeitspapier 03, Düsseldorf

Gückelhorn, C./Bispinck, R./Dribbusch, H./Öz, F. (2010a): Was verdienen Diplomkaufleute? Eine Analyse von Einkommensdaten auf Basis der WSI-Lohnspiegel-Datenbank, Arbeitspapier 06, Düsseldorf
Gückelhorn, C./Bispinck, R./Dribbusch, H./Öz, F. (2010b): Was verdienen Fachinformatiker/innen? Eine Analyse von Einkommensdaten auf Basis der WSI-Lohnspiegel-Datenbank, Arbeitspapier 06, Düsseldorf

Öz, F./Bispinck, R. (2008): Was verdienen Bürokaufleute? Eine Analyse von Einkommensdaten auf Basis der WSI-Lohnspiegel-Datenbank, Arbeitspapier 02, Düsseldorf

Öz, F./Bispinck, R. (2009a): Was verdienen Bankkaufleute? Eine Analyse von Einkommensdaten auf Basis der WSI-Lohnspiegel-Datenbank, Arbeitspapier 01, Düsseldorf

Öz, F./Bispinck, R. (2009b): Was verdienen Technikerinnen und Techniker? Eine Analyse von Einkommensdaten auf Basis der WSI-Lohnspiegel-Datenbank, Arbeitspapier 02, Düsseldorf 